\title{
Transient serum exposure regimes to support dual differentiation of human mesenchymal stem cells.
}

L.A. France ${ }^{1}$, C.A. Scotchford ${ }^{1}$, D.M. Grant ${ }^{1}$, H. Rashidi ${ }^{2}$, A.A. Popov ${ }^{2}$, V. Sottile ${ }^{2 *}$.

1 Division of Materials, Mechanics and Structures, Faculty of Engineering, University of Nottingham, Nottingham, NG7 2RD, UK

${ }^{2}$ Wolfson Centre for Stem Cells, Tissue Engineering and Modelling (STEM), School of Clinical Sciences, University of Nottingham, Nottingham, NG7 2RD, UK

\section{*Corresponding author: $\quad$ Dr Virginie Sottile}

Wolfson Centre of Stem cell, Tissue Engineering and Modelling (STEM)

CBS Building, University Park

The University of Nottingham

Nottingham NG7 2RD

United Kingdom

Tel: +44(0)1158231235 Fax: +44(0)1158468002

Email: virginie.sottile@nottingham.ac.uk

Running title: Single transient serum exposure approach to support dual MSC fate

Keywords: Mesenchymal stem cells, differentiation, serum-free medium, osteogenesis 


\begin{abstract}
Human mesenchymal stem cells (MSCs), which can generate both osteoblasts and chondrocytes, represent an ideal resource for orthopaedic repair using tissue engineering approaches. One major difficulty for the development of osteochondral constructs using undifferentiated MSCs is that serum is typically used in culture protocols to promote differentiation of the osteogenic component, whereas existing chondrogenic differentiation protocols rely on the use of serum-free conditions. In order to define conditions which could be compatible with both chondrogenic and osteogenic differentiation in a single bioreactor, we have analysed the efficiency of new biphasic differentiation regimes based on transient serum exposure followed by serum-free treatment.

MSC differentiation was assessed either in serum-free medium or with a range of transient exposure to serum, and compared to continuous serum-containing treatment. Although osteogenic differentation was not supported in the complete absence of serum, marker expression and extensive mineralisation analyses established that 5-day transient exposure triggered a level of differentiation comparable to that observed when serum was present throughout. This initial phase of serum exposure was further shown to support the successful chondrogenic differentiation of MSCs, comparable to controls maintained in serum-free conditions throughout. This study indicates that a culture based on temporal serum exposure followed by serum-free treatment is compatible with both osteogenic and chondrogenic differentiation of MSCs. These results will allow the development of novel strategies for osteochondral tissue engineering approaches using MSCs for regenerative medicine.
\end{abstract}




\section{Introduction}

In the natural joint, articular cartilage plays an important role protecting the subchondral bone from high stresses, as well as reducing nominal contact pressure and allowing low-friction movements (Mow et al., 1993; Little, 1969). Both the articular cartilage and the underlying subchondral bone are often subjected to structural damage as a result of trauma and disease, leading to severe pain, joint deformity, loss of motion and the need for surgical procedures (Tampieri et al., 2008; Swieszkowski et al., 2007). Due to the avascular, non-innervated nature of cartilage, and its limited capacity to repair itself, there is an increased demand to engineer osteochondral grafts that overcome the problems of standard chondral grafts such as donor site morbidity and poor anchorage between the native tissue and the chondral construct (Cancedda et al., 2003). Typical osteochondral grafts consist of a superficial cartilaginous layer and an underlying calcified tissue, representing cartilage and bone respectively, which often delaminate when subjected to mechanical loads and stresses of the body (Martin et al., 2007). Approaches considered for joint tissue repair include cell-free composites, eg ceramic-based devices, and scaffolds loaded with a single cell type or with more complex multi-lineage cell populations (Tampieri et al., 2008; Vinatier et al., 2009). Although more physiologically relevant, scaffolds engineered to host multiple cell types are technically challenging to seed and populate homogenously. An optimal solution would be to engineer a homogeneous scaffold using a single cell source that has both osteogenic and chondrogenic differentiation potential, thus overcoming complications of multi-cellular and multi-media approaches that can lead to delamination of the bone and cartilage regions, and ultimately contribute largely to the failure of the construct.

Mesenchymal stem cells (MSCs) have been utilised in many tissue engineering applications for several years (Caplan and Bruder., 2001), due to their ability to differentiate towards multiple lineages including osteoblasts, chondrocytes, and adipocytes (Pittenger et al., 1999; Mackay et al., 1998; Young et al., 1998). MSCs are the primary cell source for endochondral and intramembranous bone formation in vivo (Caplan et al., 1987), making them an ideal resource for the production of cell-seeded osteochondral constructs that can be utilised for bone repair. In addition, MSCs represent a potential autologous cell source, which would overcome graft rejection issues previously seen in the repair of chondral defects (Csaki et al., 2008; Bruder et al., 1994; Deans and Moseley., 2000), and autologous MSCs have already been successfully used in a clinical setting for bone repair (Quarto et al., 2001). Conditions for the osteogenic and chondrogenic differentiation of MSCs have been described for many years in the literature (Bruder et al., 1994; Bruder et al., 1997; Pittenger et al., 1999; 
Sottile et al., 2002). One key difference in the culture conditions used to stimulate osteogenic and chondrogenic differentiation is the use of serum: osteogenic treatments typically include $10 \%$ serum, whereas chondrogenic differentiation protocols are carried out in serum-free medium. The presence of serum, an ill-defined cocktail of growth factors, proteins, toxin scavengers and other source-dependant substances (Liu et al., 2007), is thus considered an unsuitable component to support the simultaneous differentiation into these two lineages.

In order to design treatment regimes which could resolve this incompatibility and provide a basis for the differentiation of both osteogenic and chondrogenic lineages in MSC-seeded constructs cultured in a single chamber, we investigated the use of new biphasic treatments. The research presented here examines a new approach to MSC differentiation using culture conditions involving transient FCS exposure, and reports on the efficacy of these new treatments for the differentiation of MSCs towards osteogenic and chondrogenic lineages. 


\section{Materials and methods}

All reagents were purchased from Invitrogen (Paisley, UK) unless otherwise stated.

\subsection{Cell culture}

A culture of an human bone marrow-derived mesenchymal stem cell (hMSCs) line (Okamoto et al., 2002; Sottile et al., 2003) was maintained in a monolayer culture in complete Dulbecco's Modified Eagle Medium (DMEM) supplemented with 10\% (v/v) foetal calf serum (FCS), $1 \%$ (v/v) L-glutamine, $1 \%$ (v/v) non-essential amino acids (NEAA) and $1 \%(\mathrm{v} / \mathrm{v})$ penicillin/ streptomycin, in a humidified atmosphere at $37^{\circ} \mathrm{C}, 5 \% \mathrm{CO}_{2}$. For the purposes of the work presented here, this medium will be referred to as standard culture medium (SC). Monolayer cultures were passaged by enzymatic digestion using $0.02 \%$ trypsin/ EDTA, producing a single cell suspension, and cells were passaged using 1 in 4 dilutions for culture maintenance. Live cell counts carried out using Trypan blue exclusion were used to determine the final cell seeding density of $\left(2 \times 10^{4}\right.$ cells/ ml $)$. For mineral deposition analysis, Thermanox ${ }^{\mathrm{TM}}$ tissue culture plastic discs (Nunc ${ }^{\mathrm{TM}}$, Fisher Scientific, UK) (13mm diameter) were placed into 24 well plates and sterilised under UV for an hour. Wells were washed with phosphate buffered saline (PBS) (OXOID, UK) and $1 \mathrm{ml}$ of standard MSC culture medium before $1 \mathrm{ml}$ MSC cell suspension was added $\left(2 \times 10^{4}\right.$ cells/ ml). Cells were allowed to attach to the Thermanox ${ }^{\mathrm{TM}}$ discs in SC for 24 hours before starting the differentiation experiment.

\subsection{Osteogenic differentiation}

To evaluate different culture conditions on the osteogenic differentiation, two medium preparations were tested: (i) OS+FCS (serum-containing osteogenic medium): DMEM supplemented with $10 \%$ (v/v) foetal calf serum (FCS), $1 \%(\mathrm{v} / \mathrm{v})$ L-glutamine, $1 \%(\mathrm{v} / \mathrm{v})$ NEAA, $1 \%$ (v/v) penicillin/ streptomycin, dexamethasone $(0.1 \mu \mathrm{M})$, ascorbic acid phosphate $(50 \mu \mathrm{M})$, and $\beta$-glycerophosphate $(10 \mathrm{mM})$ (Sigma-Aldrich) (Sottile et al. 2002) ; (ii) OS-FCS (osteogenic supplement only): recipe as stated in (i) but with no FCS. Cells (passage 37) were treated with OS+FCS for 1, 2, 3, 4 and 5 days, before switching to OS-FCS for the remainder of the 21 day culture period. Control wells were treated in parallel with OS+FCS or OS-FCS for the 21 days without switching. An additional control culture was also treated with SC medium in the absence of osteogenic supplements for the duration of the assay. A summary of the different culture conditions used is presented in figure 1a. The medium was changed every day for the first 5 days of culture, after which the medium was changed every 3 days. 


\subsection{Chondrogenic differentiation}

To determine the influence of serum exposure on the chondrogenic differentiation of hMSCs, two media were tested, (i) CS-FCS (serum-free chondrogenic medium): DMEM supplemented with 1\% (v/v) L-glutamine, $1 \%$ (v/v) NEAA, $1 \%$ penicillin/ streptomycin, dexamethasone $(0.1 \mu \mathrm{M})$, ascorbic acid phosphate $(50 \mu \mathrm{M})$, sodiumpyruvate $(1 \mathrm{mM})$, L-proline $(40 \mu \mathrm{g} / \mathrm{ml})$, TGF- $\beta(10 \mathrm{ng} / \mathrm{ml})$ and ITS+ (Sigma-Aldrich). (ii) CS+FCS (chondrogenic medium containing FCS): recipe as stated in (i) with the inclusion of $10 \%$ (v/v) foetal calf serum. Cell pellets were formed with $2.5 \times 10^{5}$ cells (passage 39) and cultured in a polystyrene falcon tube as previously described (Sottile et al., 2002). One set of pellets was treated with CS+FCS for 5 days and then switched to CS for the remainder of the 21-day culture period ( $\mathrm{CS}_{5}{ }^{\circ}$ ), and another set was treated for the 21 days with CS+FCS medium ('CS+FCS'). Pellets maintained for 21 days in CS-FCS medium ('CS-FCS') or SC medium in the absence of all chondrogenic supplements ('SC') were used to provide positive and negative controls, respectively. A summary of the different culture conditions used is presented in figure $1 \mathrm{~b}$. The medium was changed every day for the first 5 days of culture, then every 3 days for the remainder of the 21-day period.

\subsection{Alamar Blue}

Cell metabolic activity was evaluated using an Alamar blue assay after 21 days culture. This assay is a nonendpoint, non-toxic assay that measures mitochondrial metabolic activity. After the 21-day culture, the medium was removed and the samples washed 3 times with PBS at ca. $37^{\circ} \mathrm{C}$. Five hundred microlitres of Alamar Blue solution (AbD Serotec, UK) (1:10 Alamar Blue: warm Hanks Balanced Salt Solution (HBSS)) were added to each well. Three empty wells were filled as blanks, and the plate incubated for $80 \mathrm{~min}$ at $37{ }^{\circ} \mathrm{C}, 5 \% \mathrm{CO}_{2}$, then wrapped in aluminium foil and put on a plate shaker for 10 min. A $100 \mu$ l extract from each sample well was transferred to a 96-well plate in triplicate. Fluorescence was measured at 530/25 nm excitation, 590/35 nm emission using a Bio-TEK FLx800 micro-plate fluorescence reader.

\subsection{Hoechst 33258 DNA Assay}

Cell proliferation was determined by assaying for total DNA content after the 21-day culture period. The fluorochrome Hoechst 33258 (Sigma-Aldrich) binds cellular DNA resulting in enhanced fluorescence, which is directly proportional to the DNA content (Rago et al., 1990). To lyse the cells, the culture medium was replaced with $1 \mathrm{ml}$ sterile $\mathrm{dH}_{2} \mathrm{O}$, and the well plate stored at $-20{ }^{\circ} \mathrm{C}$. Once frozen, the well plate was placed into the 
incubator at $37^{\circ} \mathrm{C}$ to thaw, and the cycle repeated 3 times. Aliquots of $100 \mu \mathrm{l}$ from each well were transferred to a 96-well plate, along with $100 \mu \mathrm{l}$ Hoechst stain used at a working dilution of 1:50 in TNE buffer (10mM Tris (hydroxymethyl)methylamine, $1 \mathrm{mM}$ EDTA and $2 \mathrm{mM} \mathrm{NaCl}$ in distilled water, $\mathrm{pH} 7.4$ ). The plate was gently agitated and fluorescence measured at $360 \mathrm{~nm}$ excitation, $460 \mathrm{~nm}$ emission using a Bio-TEK FLx800 microplate fluorescence reader. A standard curve of DNA was produced using known concentrations of DNA from calf thymus (Sigma-Aldrich) reconstituted in $0.01 \mathrm{M} \mathrm{NaCl}$ at a concentration of $20 \mu \mathrm{g} / \mathrm{ml}$.

\subsection{DMMB assay}

Binding of 1, 9-dimethylmethylene blue (DMMB) to sulphate and carboxylate groups was used to quantify glycosaminoglycans (GAG) (Farndale et al., 1982). Briefly, samples were incubated with 1 mL papain solution overnight in a waterbath at $60{ }^{\circ} \mathrm{C}(0.1 \%$ papain (Sigma-Aldrich) : papain buffer (sodium phosphate $0.1 \mathrm{M}$, cysteine hydrochloride 0.005 M, EDTA 0.005 $\mathrm{M}$ and $\mathrm{dH}_{2} \mathrm{O}$ )). Papain-digested pellet samples were diluted 1:100 with heat-treated papain solution and aliquots of $20 \mu \mathrm{l}$ were taken in triplicate from each well and transferred to a 96 well-plate. A GAG reference calibration curve was generated using chondroitin-4-sulfate (Sigma-Aldrich) and $20 \mu \mathrm{L}$ DMMB solution were added to each well before optical density measurement at $540 \mathrm{~nm}$ (Bio-TEK ELx800 micro-plate reader).

\subsection{Safranin-O staining}

After 21 days culture, the pellets were washed in PBS and fixed in $4 \%$ PFA. After washing again in PBS, they were submerged into $15 \%$ sucrose in PBS for several hours, and transferred to a $30 \%$ sucrose solution and left over night. Pellets were then embedded in an OCT compound (Tissue-Tek, USA) and frozen. Samples were loaded into the cryostat and cut into sections $5 \mu \mathrm{m}$ thick and collected on SuperFrost ${ }^{\circledR}$ glass slides (VWR). Pellet sections were rinsed in PBS to remove OCT prior to being washed with $\mathrm{dH}_{2} \mathrm{O}$ and rehydrated through a series of methanol $(100 \%[\mathrm{v} / \mathrm{v}], 90 \%[\mathrm{v} / \mathrm{v}]$ and $70 \%[\mathrm{v} / \mathrm{v}])$ and rinsed in tap water. Sections were then incubated in Mayer's hematoxylin for 5 minutes, rinsed under running water to blue nuclei and rinsed in $\mathrm{dH}_{2} \mathrm{O}$. Slides were then immersed into $0.1 \%$ Safranin-O for 10 minutes and dehydrated through an increasing series of methanol $(70 \%[\mathrm{v} / \mathrm{v}], 90 \%[\mathrm{v} / \mathrm{v}]$ and $100 \%[\mathrm{v} / \mathrm{v}]$. Sections were finally cleared in xylene and mounted using DPX prior to viewing under a phase contrast microscope.

\subsection{Reverse Transcription-Polymerase Chain Reaction (RT-PCR)}


RT-PCR analysis was performed following protocols described elsewhere (Sottile et al., 2003), using the following primer sequences (Eurofins MWG, Germany): bone/liver/kidney alkaline phosphatase $(A L P)$ : forward 'gcagccactgagcgttc' / reverse 'gtgggagtgcttgtatctc' ; osteocalcin $(O C N)$ : forward 'ctcacactcctcgccctatt' / reverse 'aactcgtcacagtccggattg' ; clathrin $(C L A T H)$ : forward 'ttataatgggggaaaacagg' / reverse 'ttgtctttgttggcactga'. In brief, PCR reactions were heated at $95^{\circ} \mathrm{C}$ for $5 \mathrm{~min}$, before undergoing cycles of $\left[95^{\circ} \mathrm{C}\right.$ for $30 \mathrm{~s}, 60{ }^{\circ} \mathrm{C}$ for $45 \mathrm{~s}$ and $72{ }^{\circ} \mathrm{C}$ for $\left.1 \mathrm{~min}\right]$ repeated 30 times for $A L P$ and $C L A T H$, and 38 times for $O C N$. Expression of each marker gene was measured using the Aida Image Analyzer software and normalised against CLATH expression, used as loading controls as previously described (Sottile et al., 2002, Sottile et al., 2003).

\subsection{ELISA for osteocalcin}

An osteocalcin ELISA kit (Invitrogen, UK) was used to measure intact osteocalcin concentrations following manufacturer's instructions. The absorbance of the samples (proportional to the human osteocalcin concentration) was read at $450 \mathrm{~nm}$ using a colourmetric plate reader (Bio-TEK Elx800 micro-plate reader).

\subsection{Immunohistochemistry}

Cells were fixed in ice cold 4\% PFA for 15 min and washed in PBT (PBS $+0.1 \%$ Tween20) for 5 min. Nonspecific binding was blocked by incubation with $0.75 \%$ FCS in PBT for 1 hour. Cells were then exposed to the primary antibody (bone alkaline phosphatase and osteonectin antibodies from DSHB, osteocalcin antibody from Abcam) at a dilution of 1:200 in PBT, and stored at $4{ }^{\circ} \mathrm{C}$ overnight. After washing 3 times in PBT for 20 min intervals, cells were incubated with a 1:200 dilution of Texas Red-conjugated secondary antibody (VectorLabs) for 1 hour in the dark, before washing 4 times in PBS for 15 min per wash. Samples were finally mounted onto microscope slides using Vectashield mounting medium containing dapi (VectorLabs), and analysed with fluorescent light microscopy.

\subsection{Alizarin red staining and quantification}

After 21 days in culture, cells were fixed with $4 \%$ paraformaldehyde (PFA) for 15 min. After washing three times with PBS, $1 \mathrm{ml}$ of $1 \%$ Alizarin red (aqueous) (Sigma-Aldrich) was added to each well and left for $10 \mathrm{~min}$, prior to multiple washes with $\mathrm{dH}_{2} \mathrm{O}$ (Tataria et al., 2006). Once the excess stain had been removed, bone nodules were viewed using a phase contrast microscope. Quantitative analysis of alizarin red staining was achieved by the extraction of the stain using a de-stain solution (Tataria et al., 2006) (20\% methanol, 10\% acetic 
acid in $\mathrm{H}_{2} \mathrm{O}$ ) for $15 \mathrm{~min}$, whilst the well plates were gently agitated using a plate shaker. Alizarin red concentrations were determined using a colourmetric plate reader at a wavelength of $405 \mathrm{~nm}$ (Bio-TEK Elx800 micro-plate reader). Values obtained were normalized to DNA content/well.

\subsection{ESEM and EDX -mineralisation identification and quantification}

Cell cultures were washed 3 times with warm PBS and fixed in 3\% glutaraldehyde in $0.1 \mathrm{M}$ cacodylate buffer for $30 \mathrm{~min}$, then washed in $0.1 \mathrm{M}$ cacodylate buffer containing $7 \%$ sucrose. Cells were then viewed and analysed in a Philips FEG Environmental SEM (ESEM), operated at 10kV, with field emission gun in wet mode, allowing analysis to be carried out on un-processed samples. Resulting Energy-dispersive X-ray (EDX) spectra were used to identify elements present as a result of matrix mineralisation.

\subsection{FIB-TEM}

FIB-SEM (for lift-out) and FIB-TEM (for evaluation) were carried out using protocols detailed elsewhere (Edwards et al., 2009). In brief, prior to sectioning using an FEI Quanta 200 3D FIBSEM, samples were fixed in ice-cold 4\% PFA and dehydrated. A small strip of $\mathrm{Cu}$ conducting tape was put on the surface of the sample to provide a conductive path from a region adjacent to the region to be milled and the sample support. Ion beam induced deposition of $\mathrm{W}$ on the surface connected the $\mathrm{Cu}$ tape to the region to be milled, whilst a further $\mathrm{W}$ deposition using a gas injection system provided a $\sim 2 \mu \mathrm{m}$ thick protective layer over the region to be sectioned. A section was cut from the sample and transferred using an Omniprobe manipulator needle to a $\mathrm{Cu}$ TEM support grid for final thinning using lowering current steps, finishing at $30 \mathrm{kV}$ and $30 \mathrm{pA}$. Electron-transparent specimens were then examined using STEM (Scanning Transmission Electron Microscopy) imaging and EDX analysis, both carried out on a JEOL 2100F TEM.

\subsection{Statistics}

All experimental data represent mean \pm SEM of two repeats of experiments, with 4 wells/pellets seeded for each assay. Triplicates from each well were taken for quantitative assays. Statistical comparisons for all experimental sets were based on one-way ANOVA using Tukey's post-test for pair-wise comparisons with a $p<0.05$ considered as significant unless otherwise stated. 


\section{Results}

\subsection{Transient serum exposure supports MSC metabolic activity}

To evaluate the effects of varying FCS exposure regimes on OS-treated MSC cultures (figure1a), we performed a proliferation assay comparing the DNA content present in cultures (figure 2a). Proliferation was increased with prolonged exposure to serum in the culture media. There was no significant difference between cells maintained in $\mathrm{SC}$ medium, and $\mathrm{OS}_{0}$ cells that were not exposed to FCS. There was also no significant difference in the DNA content from cultures $\mathrm{OS}_{1}, \mathrm{OS}_{2}, \mathrm{OS}_{3}$ and $\mathrm{OS}_{4}$. However, a significant increase was observed after 5 days exposure to FCS in the $\mathrm{OS}_{5}$ culture $(p<0.05)$, which in turn showed no significant difference to $\mathrm{OS}_{21}$ $(p>0.05)$. In addition, the metabolism in MSCs cultured under OS conditions with varying FCS exposure periods was measured using the alamar blue assay (figure $2 \mathrm{~b}$ ). The development of fluorescence represented increased metabolic activity, indicating the cells were healthy and viable for proliferation. Alamar blue reduction increased with exposure to FCS, and was significantly higher in all conditions compared to the SC conditions $(p<0.001)$. No significant difference was detected between $\mathrm{OS}_{0}$, and the cultures exposed to FCS for 1,2 and 3 days. There was a significant increase in metabolic rate after 4 days exposure, but no significant difference was found for exposures longer than 4 days $(p>0.05)$. Analysis of cell viability by propidium iodide staining confirmed that MSCs were not adversely affected when exposed to FCS for 5 days compared to standard FCS-containing OS treatment (Suppl. Fig.1).

\subsection{Transient exposure to FCS supports expression of osteogenic markers}

Expression of the osteogenic markers bone-specific alkaline phosphatase $(A L P)$ and osteocalcin $(O C N)$ was analysed using RT-PCR (figure 3a). The expression of $A L P$ was higher in all conditions containing OS supplements compared to the $\mathrm{SC}$ control. Although there was no difference between $\mathrm{OS}_{0}, \mathrm{OS}_{1}, \mathrm{OS}_{2}$, exposure to FCS for 4 days or more saw a significant increase of the $A L P$ signal, with an expression level observed for 5-day exposure to FCS comparable to that observed for $\mathrm{OS}_{21}$. $O C N$ upregulation was also seen with increased exposure to FCS. While no clear difference was seen between $\mathrm{OS}_{0}$ and 1-day exposure to FCS, both showing low levels of $O C N$, a significant increase was seen after 4 days exposure to FCS with no substantial difference between $\mathrm{OS}_{4}, \mathrm{OS}_{5}$, and $\mathrm{OS}_{21}$ conditions.

To confirm this result, the concentration of OCN protein present in selected cultures was quantified using an ELISA assay (figure 3b). A significant increase in OCN concentrations was seen with increased exposure to 
FCS. There was no significant difference detected between 5 days exposure to FCS and $\mathrm{OS}_{21}(p>0.05)$. Comparable to previous data, there was a significant difference between this and $\mathrm{OS}_{0}$ which was not exposed to FCS $(p<0.001)$, which in turn was expressing higher levels of OCN than the SC control. Trace amounts of OCN protein were detected in the SC control.

Immunohistochemical staining was used to further assess presence of alkaline phosphatase (ALP), osteocalcin (OCN) and osteonectin (OSN) in hMSC cultures (figure 3c). Staining was observed for all three markers in conditions exposed to OS supplement, with a qualitative increase in expression with increased exposure to FCS. A strong ALP, OCN and OSN signal was detected in $\mathrm{OS}_{5}$ and $\mathrm{OS}_{21}$. In comparison, expression was much lower is conditions treated without FCS, but still higher than the SC control. Minimal expression ALP and OCN expression was detected in the SC control.

\subsection{Transient serum exposure supports mineral deposition}

To further evaluate the effect of transient serum exposure on MSC differentiation, cultures were stained with Alizarin red to analyse mineral deposition. The SC control showed only minimal background staining. In the presence of OS supplements, nodular aggregates were visible in all conditions, indicating the presence of calcium phosphate deposits resulting from a calcifying extra-cellular matrix (ECM) (figure 4). Quantification of the Alizarin red staining in parallel cultures showed that there was no significant difference between $\mathrm{OS}_{0}$ and $\mathrm{OS}_{1}, \mathrm{OS}_{2}$ and $\mathrm{OS}_{3}(p>0.05)$. However, a significant increase was seen after 4-day exposure to FCS $(p<0.001)$, and after a 5-day FCS exposure there was no significant difference compared to the $\mathrm{OS}_{21}$ positive control.

In order to better characterise the mineral deposited in the cultures, a more detailed microscopy analysis was performed using ESEM (figure 5). All cells treated with OS, with and without serum, displayed an enlarged stellate structure, characteristic of osteogenically differentiated MSCs compared to the SC control, which retained the small, elongated fibroblastic shape typical of undifferentiated MSCs. All samples except the negative control (SC) showed evidence of matrix mineralisation when analysed using ESEM (figure 5a). Two distinct crystal phases of the mineral were present, one visually characteristic of crystalline HA (dendritic structure), and the other of amorphous HA (spherical, globular structure). Further evaluation using EDX analysis confirmed that the matrix was rich in calcium phosphate, giving $\mathrm{Ca}: \mathrm{P}$ ratios ranging from 1.53 to 1.59 (figure 5b).

FIB-TEM was used to examine the composition of the ECM of the multilayered cell culture close to the Thermanox $^{\mathrm{TM}}$ disc, to ensure that matrix mineralisation was taking place throughout the culture, and not just on 
the surface (figure 6). The four predominant elements detected by EDX mapping of the STEM section were found to be carbon, calcium, oxygen and phosphorous. Carbon and oxygen were identified to spatially occupy the same area, whilst calcium and phosphate were shown to be co-localised (figure 6b). The carbon and oxygen signals are typical of general organic cellular matter. Trace amounts of oxygen were also detected in the same regions as the calcium and phosphorus, suggesting regions of mineralised matrix.

\subsection{Transient serum exposure is compatible with chondrogenic differentiation}

To assess whether the short serum exposure used in the above described protocol would be compatible with chondrogenesis, we performed a micromass pellet chondrogenesis assay. hMSCs were subjected to the chondrogenesis protocol in standard serum-free conditions (CS), or following a 5-day transient serum exposure $\left(\mathrm{CS}_{5}\right)$. In both cases, hMSCs showed a similar level of chondrogenic response, as shown by the measurement of GAG production (figure 7a) and by strong Safranin-O staining (figure 7b). In comparison, hMSC pellets treated in the continuous presence of serum $\left(\mathrm{CS}_{21}\right)$ failed to differentiate and remained negative, as was observed for hMSCs maintained in control medium devoid of chondrogenic cues (SC). 


\section{Discussion}

In this study, we have investigated the effect of reduced exposure to foetal calf serum on the differentiation of human mesenchymal stem cells. Although efficient differentiation was not achieved in the absence of serum throughout the treatment, our findings demonstrate that it is possible to achieve osteogenic differentiation, producing mineralising osteoblast-like cells, without FCS continuously present in the osteogenic culture medium. These results support other studies suggesting that MSC cultures can be maintained in reduced serum conditions (Gronthos and Simmons 1995; Kuznetsov et al., 1997). Although serum-free formulations for the maintenance of MSC cultures are the subject of ongoing investigations (Sotiropoulou et al., 2006; Berger et al., 2006), existing studies indicate that human bone marrow stromal cells (BMSC) demonstrated more rapid proliferation in FCS-containing medium, while little proliferation occurred in serum-free conditions, suggesting that the presence of high FCS was more effective than serum-free solutions (Kuznetsov et al., 2000). Results from our cellular assays also showed that the efficacy of the cultures with regards to proliferation and metabolism was greater in FCS-containing medium; however the use of a 5-day boost with FCS followed by serum-free medium for the remaining culture period produced results with no significant difference compared to FCS exposure for the full 21-day period. The metabolic activity and proliferation rates of the $\mathrm{OS}_{0}$ culture were still higher than the SC control, but significantly lower than those exposed to $\mathrm{OS}_{21}$, in agreement with studies showing that cells still proliferate without serum in the medium but often experience senescence, lowering the overall proliferation rates (Agata et al., 2009).

With regards to osteogenic differentiation, the literature is unclear regarding serum-free treatments of MSCs without the use of serum-replacements. Montzka et al. investigated the effect of reducing the serum content of the osteogenic media to $2 \%$ from the standard $10 \%$, and found osteogenic differentiation to be successful without the need for additional growth factors to the culture media (Montzka et al., 2010). It remained so far unclear whether temporally reducing exposure to serum would enable successful differentiation. Our study indicates that with 5-day exposure to FCS, efficient differentiation is obtained, with no significant difference for both mineralisation and osteogenic marker expression when compared to cultures with serumcontaining osteogenic medium for the complete 21-day period. This underlines the important role of FCS in the initiation process, and indicates that differentiation is not solely dependent on osteogenic supplement (ascorbic acid, dexamethasone and $\beta$-glycerophosphate). 
Bone specific alkaline phosphatase plays a major role in bone calcification, specifically matrix mineralisation and therefore its expression during osteogenic differentiation is considered to be a useful marker of successful matrix mineralisation, indicating the presence of osteoblasts-like cells (Siffert, 1951). Alkaline phosphatase expression was significantly lower in the serum-free conditions, and increased with exposure to FCS, fitting closely with the data from cell proliferation and metabolism assays. Similar results produced by Agata et al. show that ALP activity in serum-free expanded BMSC was significantly lower than that of BMSC expanded with serum, regardless of the duration of the osteogenic induction (Agata et al., 2009). In combination with the proliferation data from the current study, this suggests that $\mathrm{OS}_{0}, \mathrm{OS}_{1}$ and $\mathrm{OS}_{2}$ conditions may lead to many cells remaining in an undifferentiated state within the culture, explaining the low levels of ALP produced. Similar observations were reported when Friedman et al. found that lower ALP expression was seen in serumfree conditions before the addition of extra growth factors such as BMP to the culture medium (Friedman et al., 2006). During the late stages of osteogenic differentiation before the initiation of mineral deposition, ALP expression peaks and is usually followed by an increase in the expression of OCN, which occurs concurrently with matrix deposition and is therefore tightly associated with mineralisation (Lian et al., 1999). The trend observed for OCN expression is similar to that seen for ALP expression, confirming that the production of osteoblast-like cells is higher in the cultures exposed to FCS for longer, and that a 5-day boost gives statistically similar data to $\mathrm{OS}_{21}$ culture conditions. This suggests that there may be many cells in an undifferentiated state remaining in the conditions with minimal or no exposure to FCS.

Interestingly, it was previously reported that when bone marrow stromal cells pre-cultured in serumcontaining medium were trypsinised and treated with osteogenic supplement without FCS for 3 days before transplantation, bone formation was sustained in vivo when compared to cultures treated with serum-free medium throughout, suggesting that biphasic culture approaches for osteogenic stimulation would be compatible with efficient differentiation in vivo (Kuznetsov et al., 2000). Our observations further indicate that both proliferation rates and osteogenic differentiation can be maintained in vitro to levels similar to FCS-containing conditions if the serum boost is exercised for 5 days before switching to serum-free conditions. Analysis of the $\mathrm{Ca}^{2+}$ incorporation into the extracellular matrix with Alizarin red (Titorencu et al., 2007) confirmed that 5-days FCS inclusion in the treatment successfully produced a heavily mineralised matrix made up of calcium phosphate at the day21. It will be interesting to further evaluate whether a similar outcome can be achieved at shorter time-points, such as day14, in order to further optimise the differentiation protocol. Although the complete exclusion of FCS from OS treatment produced some calcium deposits, quantification showed that 
mineralisation was significantly lower, in agreement with data from RT-PCR, ELISA and immunostaining suggesting that little mineralisation was achieved in the absence of any serum exposure. In addition to the presence of calcium nodules confirmed by staining, ESEM revealed two different phases of the mineralised matrix in MSC cultures: a visually crystalline phase with a sharp dendritic structure, and a globular noncrystalline phase. The use of EDX confirmed that these nodules were made up of calcium phosphate with Ca:P ratios were close to that of hydroxyapatite $(\sim 1.67)$, indicating that osteogenic differentiation and successful matrix mineralisation had been achieved with transient serum treatment.

Furthermore, our results show that the inclusion of the 5-day phase of FCS exposure supports both mineralisation and successful chondrogenic differentiation of hMSCs, indicating that temporal restriction can overcome the negative impact of long-term serum use on chondrogenic cultures (Wang et al., 2010; Lee et al., 2009; Malpeli et al., 2004). Further evaluation of this transient protocol will be useful to establish the effect of short serum exposure on the levels and kinetics of chondrogenic matrix component synthesis such as collagen II and $\mathrm{X}$. The results presented here underline the benefit of developing new approaches involving transient exposure regimes in order to support dual differentiation from a single cell source. Further improvements to this approach will seek to address remaining issues related to the use of biological products such as serum, including risks associated with possible pathogens including prions and viruses, as this will be critical for subsequent translational applications (Liu et al., 2007).

These results provide the basis for the development of a cellularised scaffold from a single material, in a single chamber, with the aim to create two distinct regions harbouring osteochondral and osteogenic lineages. The identification of a culture regime compatible with both lineages allows the design of new tissue engineering approaches, in which naive MSCs seeded on a scaffold and maintained in this biphasic culture medium can be directed towards both osteogenic and chondrogenic fates by physical (such as graduated pore size) and biochemical (such as growth factor release) cues provided locally within the scaffold. Such strategies combining a united supportive differentiation medium and localised fate regulators can enable the production of single scaffolds with gradual osteochondral properties through a natural interface, as seen in the osteochondral region of a joint. 


\section{Conclusions}

In conclusion, we have identified a culture protocol that allows successful osteogenic differentiation of human mesenchymal stem cells with osteogenic supplement but without the need for long term exposure to FCS. Our data show that it is possible to treat the cells with osteogenic supplement and FCS for 5 days, and then remove the serum, achieving a level of differentiation which is comparable to that observed in cells cultured in osteogenic supplement with FCS for the full culture period. Our study also shows that this strategy of short term serum treatment successfully maintains the chondrogenic potential of MSCs. These findings represent the first step for the development of new culture approaches aiming to achieve the combined osteogenic and chondrogenic differentiation of naive MSCs seeded in single scaffolds, in a single bioreactor, for joint repair. This strategy can now be refined and integrated with other cues provided to the cells within the scaffold itself, such as localised growth factor release or differential surface chemistry, in order to allow the biological patterning of cellularised scaffolds for the repair of small skeletal defects. 


\section{Acknowledgements}

Funding from EPSRC for this study is gratefully acknowledged. We also thank Dr. Michael Fay from Nottingham Nanotechnology and Nanoscience Centre for his technical assistance with FIB-TEM. 


\section{References}

Agata H, Watanabe N, Ishii Y, Kubo N, Ohshima S, Yamazaki M, Tojo A, Kagami H. 2009; Feasibility and efficacy of bone tissue engineering using human bone marrow stromal cells cultivated in serum-free conditions. Biochem Biophys Res Commun 382: 353-358.

Berger MG, Veyrat-Masson R, Rapatel C, Descamps S, Chassagne J, Boiret-Dupre N. 2006; Cell Culture Medium Composition and Translational Adult Bone Marrow-Derived Stem Cell Research. Stem Cells 24: 28882890.

Bruder SP, Fink DJ, Caplan AI. 1994; Mesenchymal stem cells in bone development, bone repair, and skeletal regenaration therapy. J Cell Biochem 56: 283-294.

Bruder SP, Jaiswal N, Haynesworth SE. 1997; Haynesworth, Growth kinetics, self-renewal, and the osteogenic potential of purified human mesenchymal stem cells during extensive subcultivation and following cryopreservation. J Cell Biochem 64: 278-294.

Cancedda R, Dozin B, Giannoni P, Quarto R. 2003; Tissue engineering and cell therapy of cartilage and bone. Matrix Biol 22: 81-91.

Caplan AI, Bruder SP. 2001; Mesenchymal stem cells: building blocks for molecular medicine in the 21st century. Trends Mol Med 7: 259-264.

Caplan AI, Pechak DG. 1987; 'The cellular and molecular embryology of bone formation' in: Bone and Mineral Research, ed. Peck WA), Elsevier, New York, USA; 117-184.

Csaki C, Schneider PR, Shakibaei M. 2008; Mesenchymal stem cells as a potential pool for cartilage tissue engineering. Ann Anat 190: 395-412.

Deans RJ, Moseley AB. 2000; Mesenchymal stem cells: Biology and potential clinical uses. Exp Hematol 28: 875-884.

Edwards HK, Fay MW, Anderson SI, Scotchford CA, Grant DM, Brown PD. 2009; An appraisal of ultramicrotomy, FIBSEM and cryogenic FIBSEM techniques for the sectioning of biological cells on titanium substrates for TEM investigation. J Microsc 234: 16-25.

Farndale RW, Sayers CA, Barrett AJ. 1982; A direct spectrophotometric microassay for sulfated glycosaminoglycans in cartilage cultures. Connect Tissue Res 9: 247-248.

Friedman MS, Long MW, Hankenson KD. 2006; Osteogenic differentiation of human mesenchymal stem cells is regulated by bone morphogenetic protein-6. J Cell Biochem 98: 538-554.

Gronthos S, Simmons PJ. 1995; The growth factor requirements of STRO-1-positive human bone marrow stromal precursors under serum-deprived conditions in vitro. Blood 85: 929-940.

Kuznetsov SA, Friedenstein AJ, Robey PG. 1997; Factors required for bone marrow stromal fibroblast colony formation in vitro. Br J Haematol 97: 561-570.

Kuznetsov SA, Mankani MH, Robey PG. 2000; Effect of Serum on Human Bone Marrow Stromal Cells: Ex Vivo Expansion and in Vivo Bone Formation. Transplantation 70: 1780-1787.

Lee S, Kim JH, Jo CH, Seong SC, Lee JC, Lee MC. 2009; Effect of serum and growth factors on chondrogenic differentiation of synovium-derived stromal cells. Tissue Eng Part A 15: 3401-3415.

Lian JB, Stein GS, Stein JL, van Wijnen AJ. 1999; Regulated expression of the bone-specific osteocalcin gene by vitamins and hormones. Vitam Horm 55: 443-509.

Little K. 1969; Nature of osteopetrosis. Br Med J 2: 49-50. 
Liu CH, Wu ML, Hwang SM. 2007; Optimization of serum free medium for cord blood mesenchymal stem cells. Biochem Eng J 33: 1-9.

Mackay AM, Beck SC, Murphy JM, Barry FP, Chichester CO, Pittenger MF. 1998; Chondrogenic differentiation of cultured human mesenchymal stem cells from marrow. Tissue Eng 4: 415-428.

Malpeli M, Randazzo N, Cancedda R, Dozin B. 2004; Serum-free growth medium sustains commitment of human articular chondrocyte through maintenance of Sox9 expression. Tissue Eng 10: 145-155.

Martin I, Miot S, Barbero A, Jakob M, Wendt D. 2007; Osteochondral tissue engineering. J Biomech 40: 750765.

Montzka K, Führmann T, Wöltje M, Brook GA. 2010; Expansion of human bone marrow-derived mesenchymal stromal cells: serum-reduced medium is better than conventional medium. Cytotherapy 12: 587-592.

Mow VC, Ateshian GA, Spilker RL. 1993; Biomechanics of diarthrodial joints: a review of twenty years of progress. J Biomech Eng 115: 460-467.

Okamoto T, Aoyama T, Nakayama T, Nakamata T, Hosaka T, Nishijo K, Nakamura T, Kiyono T, Toguchida J. 2002; Clonal heterogeneity in differentiation potential of immortalized human mesenchymal stem cells. Biochem Biophys Res Commun. 295: 354-361.

Pittenger MF, Mackay AM, Beck SC, Jaiswal RK, Douglas R, Mosca JD, Moorman MA, Simonetti DW, Craig S, Marshak DR. 1999; Multilineage potential of adult human mesenchymal stem cells. Science 284: 143-147.

Quarto R, Mastrogiacomo M, Cancedda R, Kutepov SM, Mukhachev V, Lavroukov A, Kon E, Marcacci M. 2001; Repair of large bone defects with the use of autologous bone marrow stromal cells. N Engl J Med 344: 385-386.

Rago R, Mitchen J, Wilding G. 1990; DNA fluorometric assay in 96-well tissue culture plates using Hoechst 33258 after cell lysis by freezing in distilled water. Anal Biochem 191: 31-34.

Siffert RS. 1951; The role of alkaline phosphatase in osteogenesis. J Exp Med 93: 415-426.

Sotiropoulou PA, Perez SA, Salagianni M, Baxevanis CN, Papamichail M. 2006; Cell Culture Medium Composition and Translational Adult Bone Marrow-Derived Stem Cell Research. Stem Cells 24: 1409-1410.

Sottile V, Halleux C, Bassilana F, Keller H, Seuwen K. 2002; Stem cell characteristics of human trabecular bone-derived cells. Bone 30: 699-704.

Sottile V, Thomson A, McWhir J. 2003; In Vitro osteogenic differentiation of human ES cells. Cloning Stem Cells 5: 149-155.

Swieszkowski W, Tuan BH, Kurzydlowski KJ, Hutmacher DW. 2007; Repair and regeneration of osteochondral defects in the articular joints. Biomol Eng 24: 489-495.

Tampieri A, Sandri M, Landi E, Pressato D, Francioli S, Quarto R, Martin I. 2008; Design of graded biomimetic osteochondral composite scaffolds. Biomaterials 29: 3539-3546.

Tataria M, Quarto N, Longaker MT, Sylvester KG. 2006; Absence of the p53 tumor suppressor gene promotes osteogenesis in mesenchymal stem cells. J Pediatr Surg 41: 624-632.

Titorencu I, Jinga VV, Constantinescu E, Gafencu AV, Ciohodaru C, Manolescu I, Zaharia C, Simionescu M. 2007; Proliferation, differentiation and characterization of osteoblasts from human BM mesenchymal cells. Cytotherapy 9: 682-696.

Vinatier C, Mrugala D, Jorgensen C, Guicheux J, Noël D. 2009; Cartilage engineering: a crucial combination of cells, biomaterials and biofactors. Trends Biotechnol. 27: 307-314. 
Wang CY, Chen LL, Kuo PY, Chang JL, Wang YJ, Hung SC. 2010; Apoptosis in chondrogenesis of human mesenchymal stem cells: effect of serum and medium supplements. Apoptosis 15: 439-449.

Young RG, Butler DL, Weber W, Caplan AI, Gordon SL, Fink DJ. 1998; Use of mesenchymal stem cells in a collagen matrix for achilles tendon repair. J Orthop Res 16: 406-413. 


\section{Figure Legends}

Figure 1: Summary of the culture conditions used for the osteogenic (a) and chondrogenic (b) differentiation assays carried out in the study, describing the sequential treatments with standard culture medium (SC), serumfree osteogenic medium (OS-FCS), serum-containing osteogenic medium (OS+FCS), serum-free chondrogenic medium (CS-FCS) and serum-containing chondrogenic medium (CS+FCS).

Figure 2: Analysis of growth kinetics in cultures maintained in the different experimental conditions over 21 days. (a) DNA content from cultures after variable exposure to OS+FCS over a 21-day period. Cell proliferation was significantly higher with an increased exposure to OS+FCS. No significant difference was found between 5 and 21 days exposure to OS+FCS. Bars represent mean \pm SEM (n=4). (b) Metabolic activity in cultures after variable exposure to OS+FCS over a 21-day period. Alamar blue reduction increased with longer exposure to OS+FCS. There was a significant increase after 4 days exposure but no significant difference thereafter. Bars represent mean $\pm \operatorname{SEM}(n=4) .\left({ }^{*} p<0.05,{ }^{* *} p<0.001\right)$. Culture conditions tested as defined in Fig. 1.

Figure 3: Analysis of osteogenic markers after variable exposure to OS+FCS for a 21-day period. (a) PCR analysis of alkaline phosphatase $(A L P)$ and osteocalcin $(O C N)$ expression at day 21 , using clathrin $(C L A T H)$ as housekeeping gene control, shows upregulation with increased exposure to OS+FCS with no significant difference detected between $\mathrm{OS}_{5}$ and $\mathrm{OS}_{21}$ conditions. (b) Osteocalcin protein quantification in cultures with variable exposure to $\mathrm{OS}+\mathrm{FCS}$ shows longer exposure to $\mathrm{OS}+\mathrm{FCS}$ leads to higher OCN expression detectable in the cell culture. No significant difference was detected between 4 days exposure to OS+FCS and 21 days exposure to OS+FCS $\left({ }^{*} p<0.05\right)$. (c) Immunostaining for alkaline phosphatase (ALP), osteocalcin (OCN) and osteonectin (OSN) expression (red) in MSCs with variable exposure to FCS, with dapi couterstain (blue). Increase in expression of all osteoblastic markers was detected with an increased exposure to OS+FCS. Minimal expression for ALP and OCN was detected in the standard control, with significant upregulation in cells treated with OS+FCS. Bar $=60 \mu \mathrm{m}$. Culture conditions tested as defined in Fig.1.

Figure 4: Alizarin Red staining of calcium containing nodules present in the mineralised matrix laid down after 21 days in culture with variable exposure to OS+FCS. (a) Wells stained from all conditions except SC control were positive for mineralisation with nodules clearly visible. (b) Micrographs of stained nodules show variances 
in the intensity of the staining, indicating an increase in mineralisation with prolonged exposure to OS+FCS. (c) Quantification of calcium nodules present in mineralised matrix after 21 days culture. Alizarin red staining was stronger with increased exposure to OS+FCS. There was a significant increase after 4 days exposure $\left(*^{*} p<0.001\right)$, but no significant difference there after $(p>0.05)$. Bars represent mean \pm SEM $(n=4)$. Culture conditions tested as defined in Fig.1.

Figure 5: ESEM analysis locating calcium phosphate depositions within the mineralised matrix after 21 days culture in control medium (SC) or osteogenic medium with variable exposure to FCS $\left(\mathrm{OS}_{5}, \mathrm{OS}_{21}, \mathrm{OS}_{0}\right)$. ESEM images (a) show mineral deposition and a change in morphology typical of osteogenically differentiated MSCs. Dendritic and globular mineral deposits visible in $\mathrm{OS}_{5}, \mathrm{OS}_{21}$, and $\mathrm{OS}_{0}$ cultures are typical structures of crystalline and amorphous hydroxyapatite respectively. (b) EDX spectra and $\mathrm{Ca} / \mathrm{P}$ ratios confirming the presence of calcium and phosphate in the mineralised matrix after 21 days culture in with variable exposure to OS+FCS. Culture conditions tested as defined in Fig.1.

Figure 6: FIB-TEM analysis of multilayered cell composition close to Thermanox ${ }^{\mathrm{TM}}$ disc, showing ECM mineralisation throughout the layers after 21 days in culture. EDX of the STEM sample (a) identified 4 predominant elements (b): carbon, calcium, oxygen, phosphorus. Carbon and oxygen were detected in same region, typically representing organic cell material, whilst calcium and phosphorus also co-localised, typically containing trace amounts of oxygen, confirming the presence of a mineralised matrix.

Figure 7: Analysis of MSCs exposed to chondrogenic treatment for 21 days. (a) GAG quantification obtained using the DMMB assay ( $n=3$ ). (b) Safranin-O staining with hematoxylin counterstaining of sections from micromass pellets grown in chondrogenic conditions with varying exposure to FCS. Bar $=140 \mu \mathrm{m}$. Culture conditions tested as defined in Fig.1. 


\section{Supplementary Material:}

\section{Suppl. methods:}

Flow cytometry analysis of propidium iodide-stained cells:

MSCs were treated in 6-well plates and harvested at day 14 using trypsin/EDTA. After centrifugation 5min at $200 \mathrm{~g}$, cells were resuspended in propidium iodide $(1 \mu \mathrm{g} / \mathrm{ml}$ in PBS), and kept in the dark until FACS analysis. Duplicate biological samples were analysed using a Coulter Altra to measure 50000 events per tube. Culture conditions tested as defined in Fig.1.

\section{Figure legend:}

Suppl. Figure 1: Flow cytometry analysis of propidium iodide-stained cells measured at day 14 showing fewer necrotic cells in $\mathrm{OS}_{5}$ treatment than in $\mathrm{OS}_{21}$. 